\title{
Autoeficacia y logro de aprendizaje en estudiantes con diferente estilo cognitivo en un ambiente m-learning ${ }^{1}$
}

\author{
Omar López-Vargas², Juliana Ortiz-Vásquez ${ }^{3}$ y Jaime Ibáñez-Ibáñez ${ }^{4}$ \\ Universidad Pedagógica Nacional, Bogotá (Colombia)
}

Recibido: 11/02/2019 Aceptado: 08/11/2019

\begin{abstract}
Resumen
Objetivo. Explorar los efectos de un andamiaje motivacional sobre el logro académico y la autoeficacia, tanto académica como online, en estudiantes con diferente estilo cognitivo en la dimensión DependenciaIndependencia de Campo (DIC), cuando aprenden contenidos matemáticos en un ambiente m-learning. Método. Participaron 56 estudiantes de educación secundaria de un colegio femenino público de la ciudad de Villavicencio, Colombia. La investigación siguió un diseño cuasi-experimental. Los participantes fueron distribuidos de forma aleatoria en dos grupos: (a) un grupo de estudiantes interactuó con un ambiente m-learning, el cual incluyó dentro de su estructura un andamiaje motivacional; y (b) otro grupo interactuó con un ambiente $m$-learning sin andamiaje. El estilo cognitivo de las estudiantes se determinó a través de pruebas y se aplicaron dos pos-test de autoeficacia. Resultados. El andamiaje favoreció tanto el logro académico como la autoeficacia académica y online de las estudiantes con diferente estilo cognitivo. Conclusión. Los datos evidenciaron que las estudiantes, en la dimensión DIC, lograron aprendizajes equivalentes debido al efecto del andamiaje motivacional que fue incluido en el ambiente $m$-learning. También fue posible establecer que tanto la autoeficacia académica como la autoeficacia online de los aprendices dependientes de campo favorecen el logro académico.
\end{abstract}

Palabras clave. Andamiaje, autoeficacia, diferencias individuales, logro académico, ambiente m-learning.

\section{Self-efficacy and Learning Achievement in Students with Different Cognitive Style in an M-learning Environment}

\footnotetext{
Abstract

Objective. To explore the effects of a motivational scaffolding on academic achievement, self-efficacy, both academic and online, in students with different cognitive style in the Field Dependence-Independence (FDI) dimension, when mathematical content is delivered in an m-learning environment. Method. Fifty-six secondary school students from a public women's school in the city of Villavicencio, Colombia participated. The research

Proyecto de investigación sobre fomento de la autonomía en el aprendizaje con escenarios computacionales, apoyado por el grupo de investigación Cognitek de la Universidad Pedagógica Nacional.

2 Doctor en Educación. Dirección de correspondencia: calle 72 \# 11-86, Bogotá, Colombia. Correo de correspondencia: olopezv@pedagogica.edu.co

3 Magíster en Tecnologías de la Información Aplicadas a la Educación.

4 Magíster en Tecnologías de la Información Aplicadas a la Educación.
} 
followed a cuasi-experimental design in which the participants were randomly distributed in two groups: (a) a group of students interacts with an m-learning environment, which includes within its structure a motivational scaffolding and; (b) another group interacts with an m-learning environment without scaffolding. The cognitive style of the students was determined through tests and two self-efficacy post-tests were applied. Results. The scaffolding favored both academic achievement and academic self-efficacy as well as the online students with different cognitive style. Conclusion. The data shows that students in the FDI dimension, achieved equivalent learning due to the effect of the motivational scaffolding that was included in the m-learning environment. It is also possible to establish that both academic self-efficacy and online self-efficacy of field dependent accessories favors academic achievement.

Keywords. Scaffolding, self-efficacy, individual differences, academic achievement, m-learning environment.

\section{Autoeficácia de aprendizagem em estudantes com diferente estilo cognitivo num ambiente m-learning}

\section{Resumo}

Escopo. Explorar os efeitos de um andaime motivacional sobre o logro académico e a autoeficácia tanto académica como online, em estudantes com diferente estilo cognitivo na dimensão Dependência-Independência de Campo (DIC), quando aprendem conteúdos matemáticos num ambiente m-learning. Metodologia. Participaram 56 estudantes de educação secundária de uma escola feminina pública da cidade de Villavicencio, Colômbia. A pesquisa seguiu um desenho quase- experimental. Os participantes foram distribuídos de forma aleatória em dois grupos: (a) um grupo de estudantes interagiu com um ambiente m-learning o qual incluiu dentro de sua estrutura um andaime motivacional; e b) um outro grupo interagiu com um ambiente m-learning sem andaime. O estilo cognitivo das estudantes foi determinado através de provas e foram aplicados dois post-test de autoeficácia. Resultados. $\mathrm{O}$ andaime favoreceu tanto o logro académico como a autoeficácia académica e online das estudantes com diferente estilo cognitivo. Conclusão. Os dados evidenciaram que as estudantes, na dimensão DIC, lograram aprendizagens equivalentes devido ao efeito do andaime motivacional que foi incluído no ambiente $m$-learning. Também foi possível estabelecer que tanto a autoeficácia académica como a autoeficácia online dos aprendizes dependentes de campo favorecem o logro académico.

Palavras-chave. Andaime, autoeficácia, diferencias individuais, logro académico, ambiente m-learning.

\section{Introducción}

El aprendizaje móvil, o m-learning, es una forma de favorecer el aprendizaje apoyado en tecnologías móviles. En estos ambientes de aprendizaje, los estudiantes pueden acceder a los materiales de estudio a través de teléfonos móviles, inteligentes o tabletas, entre otros. De tal manera que el proceso de aprendizaje se puede llevar a cabo en cualquier momento $y$ en cualquier lugar, de una forma flexible y dinámica, atendiendo a las características de portabilidad y conectividad de las herramientas utilizadas (Kukulska-Hulme y Traxler, 2005; Yorganci, 2017). El uso de estas tecnologías en el contexto escolar atrae la atención de educadores e investigadores en el ámbito de las tecnologías de la información aplicadas a la educación, debido a la masificación de las mismas durante las últimas dos décadas (Razzaq, Samiha y Anshari, 2018; Zheng, Li y Chen, 2016).

Algunos investigadores afirman que el uso de las tecnologías móviles facilita y amplía el alcance de los procesos se enseñanza-aprendizaje, por cuanto están en capacidad de favorecer el aprendizaje colaborativo, la motivación, la construcción de conocimiento y la autorregulación del aprendizaje, entre otros (Huang, Jeng y Huang, 2009; Zheng et al., 2016). Sin embargo, algunos estudios muestran que el uso efectivo y eficiente de las tecnologías móviles, por parte de los sujetos que toman cursos a través de ambientes m-learning, puede estar asociado con la percepción de autoeficacia y con el estilo cognitivo del estudiante. 
Con respecto a la autoeficacia, los estudios señalan que los juicios que realizan los estudiantes respecto de sus capacidades, para lograr un resultado deseado, se constituye en una variable asociada que puede determinar el éxito o fracaso de los objetivos esperados. La generalidad de los estudios da cuenta de una correlación positiva entre los juicios de autoeficacia académica y el logro de aprendizaje obtenido por los estudiantes (Bandura, 1977; Compeau y Higgins, 1995; Tsai, Chuang, Liang y Tsai, 2011). En el contexto de las tecnologías de la información y la comunicación (TIC), la autoeficacia se puede definir de forma general, como las creencias que tienen los estudiantes sobre sus capacidades para usar diferentes componentes informáticos, buscar datos y preparar informes a través de las tecnologías computacionales (Compeau y Higgins, 1995; Tsai y Tsai, 2010; Yang, 2012; Yorganci, 2017).

En este orden de ideas, la percepción de autoeficacia de los estudiantes hacia el uso de las TIC, para tomar cursos apoyados con tecnologías móviles, es una variable de análisis que permite comprender y explicar su conducta cuando adelantan cursos m-learning (Razzaq et al., 2018; Yang, 2012; Yorganci, 2017).

En cuanto al estilo cognitivo, por ejemplo, en la dimensión Dependencia-Independencia de Campo (DIC), la mayoría de los estudios muestran que los estudiantes independientes de campo (IC) usan de forma más eficiente los ambientes de aprendizaje basados en la web y obtienen mejores logros de aprendizaje que sus compañeros dependientes de campo (DC). En este ámbito de trabajo, las investigaciones muestran de forma sistemática que los estudiantes IC se desempeñan de forma eficiente y eficaz cuando interactúan con ambientes de aprendizaje basados en la web, en comparación con sus compañeros DC, quienes presentan algunas dificultades a la hora de navegar de manera libre y sin ningún tipo de apoyo en ambientes de tipo hipermedial (Chen y Macredie, 2001; Chou, 2001; Handal y Herrington, 2004; López-Vargas, DuarteSuárez e Ibáñez-Ibáñez, 2017; López, Sanabria y Sanabria, 2014).

En esta línea de trabajo, existen investigaciones que dan cuenta de una posible asociación entre el estilo cognitivo en la dimensión (DIC) y la percepción de autoeficacia académica en el desarrollo de tareas en ambientes de aprendizaje basados en computador. Es decir, los estudiantes IC emiten juicios positivos frente a sus capacidades para la realización de tareas académicas a través de la web, mientras que sus compañeros DC presentan dudas respecto de su desempeño en este tipo de ambientes (DeTure, 2004; López y Triana, 2013; López y Valencia, 2012; Valencia-Vallejo, LópezVargas y Sanabria-Rodríguez, 2018).

Además, diversos estudios indican que el uso de andamiajes puede favorecer el desempeño de los sujetos cuando emprenden tareas de aprendizaje de forma autónoma en ambientes basados en la web (Kim y Hannafin, 2011; Lehmann, Hähnlein y Ifenthaler, 2014; Zhang y Quintana, 2012). En este ámbito de investigación, el uso de andamiajes, de tipo motivacional, en escenarios online, se constituye en un apoyo para que los aprendices estén en capacidad de lograr el desempeño deseado $y$, en alguna medida, potencializa las creencias personales con respecto de sus capacidades para aprender en ambientes web, teniendo en cuenta las diferencias individuales (López y Triana, 2013; López y Valencia, 2012; Valencia-Vallejo et al., 2018).

Sin embargo, los resultados de las investigaciones en este campo no son concluyentes y no existe un acuerdo entre la comunidad académica frente a su uso potencial dentro de la estructura de los ambientes online, atendiendo a las diferencias individuales de los estudiantes. En este sentido, los estudios se encuentran en un estado preliminar. Por tanto, se hace necesario continuar con la indagación para comprender y explicar la conducta de los estudiantes de diferente estilo cognitivo cuando aprenden con tecnologías móviles.

De igual forma, es necesario realizar investigaciones sobre la percepción de autoeficacia académica y online que tengan los estudiantes de secundaria, pues dichas experiencias son escasas en este nivel educativo. Sería importante, también, establecer si el uso de tecnologías móviles representa un potencial educativo en este tipo de poblaciones, con el fin de proponer alternativas de solución que favorezcan su uso potencial para obtener logros de aprendizaje más equitativos y que respeten las diferencias individuales de los sujetos, en lo que respecta a su precepción de autoeficacia y su estilo cognitivo. 


\section{Percepción de autoeficacia en ambientes computacionales}

La autoeficacia es una noción desarrollada en la Teoría Social Cognitiva de Bandura (1997). Se puede definir como los juicios que hacen las personas sobre sus propias capacidades para organizar y ejecutar las acciones necesarias para el logro de distintos objetivos. Es uno de los indicadores motivacionales más estudiados en el contexto de la educación. Al respecto, este autor propone cuatro fuentes principales que determinan la autoeficacia de las personas: (a) las experiencias exitosas o de dominio, (b) las experiencias vicarias, (c) la persuasión verbal e influencia social, y (d) los estados fisiológicos. De estas cuatro fuentes, las experiencias exitosas constituyen la forma más eficaz de crear un fuerte sentido de eficacia personal en el estudiante.

En el ámbito educativo, los estudiantes con altos niveles de autoeficacia obtienen desempeños académicos más altos que aquellos que no confían en sus propias capacidades. Los estudiantes con estas características son capaces de gestionar, de manera más eficiente, su propio proceso de aprendizaje. Poseen una alta confianza en sus referentes internos, situación que los hace más persistentes en el logro de sus metas y, si es necesario, emplear un mayor esfuerzo en la superación de las dificultades (Bandura, 1997; Zimmerman y Kulikowich, 2016).

En el contexto de las TIC, la autoeficacia estudia las percepciones que tienen los estudiantes frente al uso de diferentes componentes informáticos, así como hacia la búsqueda de información en la web y en la preparación de informes con apoyo de tecnologías computacionales (Compeau y Higgins, 1995; Papastergiou, Gerodimos y Antoniou, 2011). En general, los estudios coinciden en afirmar que la percepción de autoeficacia hacia el uso de los computadores juega un papel importante en la decisión de las personas para utilizar internet como apoyo en los procesos de aprendizaje online. En este orden de ideas, también es posible hablar de autoeficacia online cuando los estudiantes evalúan sus percepciones de eficacia para tomar un curso en la modalidad m-learning (Razzaq et al., 2018; Tsai y Tsai, 2010; Yang, 2012; Yorganci, 2017).

Algunos estudios muestran que estudiantes con altos niveles de autoeficacia hacia las TIC, tienden a tener mejores estrategias de procesamiento de información en línea y son capaces de buscar y procesar información digital de forma estructurada.
Además, pueden comunicarse de forma eficiente con sus compañeros y profesores y muestran una actitud positiva para adelantar procesos de aprendizaje online (Aesaert, Voogt, Kuiper y Van Braak, 2017; Hatlevik, Throndsen, Loi y Gudmundsdottir, 2018; Tsai y Tsai, 2010). Aunque los resultados no son concluyentes y no existe un consenso entre la comunidad académica al respecto, se evidencia la importancia de estudiar la autoeficacia de los estudiantes cuando aprenden con tecnologías móviles (Razzaq et al., 2018; Yang, 2012; Yorganci, 2017).

\section{El estilo cognitivo en la dimensión DIC y ambientes digitales}

En el contexto educativo, el estilo cognitivo más estudiado probablemente sea el denominado Dependencia / Independencia de Campo (DIC), propuesto y desarrollado por Witkin, Moore, Goodenough y Cox (1977). En el ámbito de las TIC aplicadas a la educación, las investigaciones sobre estilo cognitivo en la dimensión DIC, muestran de forma sistemática en que los estudiantes IC obtienen mejores desempeños que sus compañeros DC, cuando interactúan con ambientes computacionales. Los estudios afirman que los estudiantes DC prefieren que el material de estudio esté organizado en forma secuencial (lineal); es decir, cuando el formato se les presenta en formato hipermedial, se desorientan fácilmente y no son capaces de tomar decisiones sobre dónde empezar y en qué dirección continuar. Por lo tanto, se les dificulta estructurar y reestructurar la información de forma eficaz durante su proceso de aprendizaje. Prefieren que el proceso de navegación en el escenario digital se realice en grupo y sea guiado por personas externas y que el control del proceso de aprendizaje sea ejercido por el propio ambiente computacional (Chen y Macredie, 2001; Handal y Herrington, 2004; López-Vargas, Ibáñez-Ibáñez y Racines-Prada, 2017).

En contraste, los estudiantes IC prefieren libertad de navegación por toda la estructura del ambiente digital, lo que hace que se desenvuelvan de forma eficaz en ambientes de tipo hipermedial. Son capaces de establecer rutas de navegación de forma organizada, no se distraen fácilmente con la información irrelevante y usan adecuadamente la mayoría de los recursos que tienen disponibles en el ambiente computacional. Asimismo, les gusta 
trabajar de forma individual (Chen y Macredie, 2001; Chou, 2001; Valencia-Vallejo et al., 2018).

En esta línea de investigación es posible relacionar de forma positiva ciertas características estilísticas de los estudiantes IC con variables motivacionales de los mismos, entre las que se destacan la orientación a metas intrínsecas y la autoeficacia (López, Hederich y Camargo, 2011). De igual manera, algunos estudios han mostrado que los aprendices IC poseen mayores niveles de autoeficacia comparados con estudiantes intermedios y DC (DeTure, 2004; López y Triana, 2013; López y Valencia, 2012; López et al., 2014).

\section{Andamiajes computacionales}

El concepto de andamiaje surge de los planteamientos de la zona de desarrollo próximo de Vygotsky (1978) y se refiere al apoyo que recibe un sujeto de otra persona para lograr una meta. Los andamiajes están diseñados para apoyar a los aprendices en el desarrollo de una tarea de aprendizaje, la cual está más allá de sus capacidades (Wood, Bruner y Ross, 1976). En las TIC, un andamiaje educativo es el apoyo que le permite al estudiante participar activamente en la ejecución de una tarea (Duffy y Azevedo, 2015). En este sentido, los andamiajes computacionales son programas de software que utilizan estrategias, tales como mensajes, notas de retroalimentación y ventanas emergentes, entre otras, para estimular la comprensión conceptual, los procesos de reflexión metacognitiva y la motivación del estudiante cuando interactúa, con escenarios computacionales (Belland, Kim y Hannafin, 2013).

En esta medida, se han diseñado andamiajes que permiten favorecer la motivación hacia el aprendizaje por parte de los estudiantes, cuando aprenden diferentes contenidos en ambientes de aprendizaje basados en computador (Alias, 2012; D’Mello, Lehman y Graesser, 2011; López et al., 2014; López y Triana, 2013; López y Valencia, 2012; Valencia-Vallejo et al., 2018).

En la presente investigación se diseñó un andamiaje de tipo motivacional dentro de un ambiente m-learning para favorecer la autoeficacia de los estudiantes con diferente estilo cognitivo en la dimensión DIC. Con base en estos planteamientos, el presente estudio se propuso dar respuesta a las siguientes preguntas de investigación:
1. ¿Cuál es el efecto de un andamiaje motivacional sobre la autoeficacia académica, la autoeficacia online y el logro de aprendizaje en estudiantes de secundaria que aprenden contenidos matemáticos en un ambiente m-learning?

2. ¿Existen diferencias significativas en la autoeficacia académica, la autoeficacia online y el logro de aprendizaje entre sujetos con diferente estilo cognitivo en la dimensión Dependencia/Independencia de Campo, cuando interactúan con un ambiente m-learning?

3. ¿Existe una interacción significativa en la autoeficacia académica, la autoeficacia online y el logro de aprendizaje, de acuerdo con las categorías del estilo cognitivo y el uso de un andamiaje motivacional en un ambiente de aprendizaje m-learning?

\section{Método}

\section{Diseño}

El estudio siguió un diseño cuasi-experimental en el que los participantes fueron asignados de forma aleatoria a dos grupos de trabajo, por lo que, de acuerdo con Campbell y Stanley (2005), se garantiza la equivalencia de los grupos. Como variable independiente del estudio se tomó el ambiente m-learning con dos valores: un ambiente m-learning que contenía un andamiaje de autoeficacia y un ambiente m-learning sin andamiaje. El estudio tuvo una variable asociada, el estilo cognitivo en la dimensión DIC con tres valores: dependiente, intermedio e independiente de campo. Las variables dependientes de la investigación fueron tres: (a) la autoeficacia académica, (b) la autoeficacia online, y (b) el logro de aprendizaje en términos de resolución de problemas de factorización (promedio de los resultados de las evaluaciones).

\section{Participantes}

Participaron 56 estudiantes de grado octavo del Colegio Nacional Femenino de Villavicencio ubicado en el departamento de Meta, Colombia. Por la naturaleza de la institución, el 100\% de las participantes fueron mujeres. La edad osciló entre los 13 y los 15 años $(M=13.44 ; D E=0.54)$. 
Los estudiantes fueron asignadas aleatoriamente a dos grupos: 28 estudiantes interactuaron con un ambiente m-learning, el cual incluyó dentro de su estructura un andamiaje motivacional, y la otra mitad de estudiantes interactuó con un ambiente $m$-learning sin andamiaje.

\section{Instrumentos}

\section{Ambiente m-learning.}

La aplicación móvil fue diseñada en la plataforma App Inventor, un entorno de desarrollo de software creado por Google Labs, para la elaboración de aplicaciones destinadas al sistema operativo Android. El software contiene el dominio de conocimiento sobre los primeros casos de factorización. En su estructura se implementó un andamiaje de tipo motivacional para facilitar el desarrollo de la autoeficacia durante el proceso de aprendizaje.

El diseño del andamiaje se fundamentó en la teoría de la autoeficacia de Bandura (1997) y los planteamientos para el diseño de andamiajes motivacionales propuestos por Belland et al. (2013) y Keller (2010). Las siguientes fueron las etapas del andamiaje:

1. Definir la tarea de aprendizaje: el ambiente m-learning ofrece una presentación de la tarea de aprendizaje (casos de factorización). De igual forma, establece las reglas y la forma como se evaluarán los aprendizajes. La intencionalidad de este escenario es generar distintas percepciones de carácter motivacional y cognitivo, atendiendo a que el estudiante puede hacerse juicios de valor sobre su efectividad personal para desarrollar la tarea (figura 1).

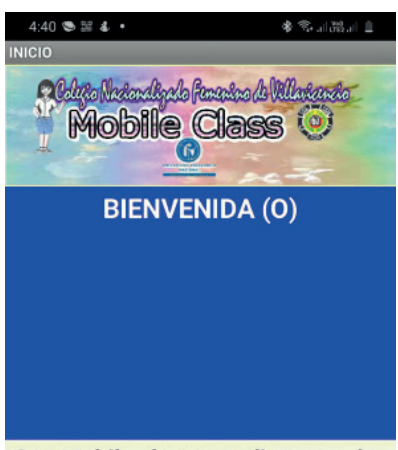

Con Mobile class estudiaremos los primeros casos de factorización, encontrarás 4 unidades :

introducción, factor común, factor común por agrupación y diferencia de cuadrados. Cada una de las unidades ofrece tres niveles de dificultad (básico, intermedio y alto). A medida que avanzas en tu estudio, pueden aparecer preguntas con el fin de comprobar tu comprensión. Debes resolverlas y si hay dudas volver a repasar III ○

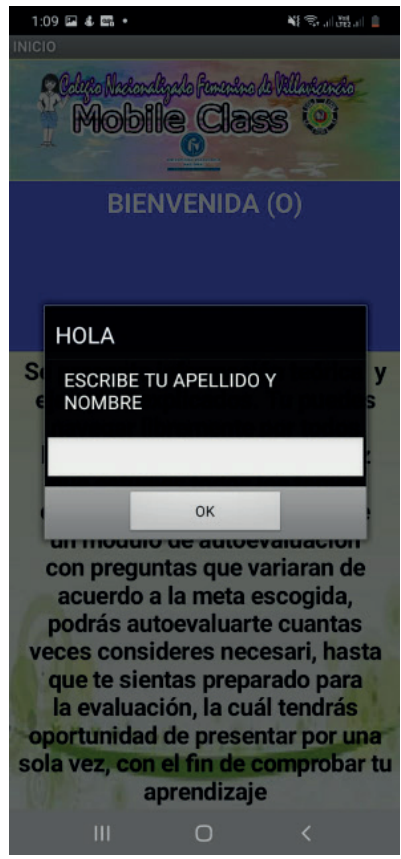

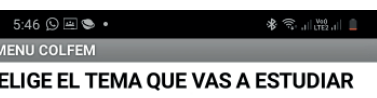

ELIGE EL TEMA QUE VAS A ESTUDIAR

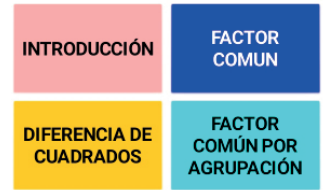

III

Figura 1. Tarea de aprendizaje elaborada en la Plataforma App Inventor, de Google Labs.

Fuente: elaboración propia.

2. Formulación de metas: la estudiante debía tomar decisiones para fijar una meta de aprendizaje y realizar un plan estratégico que le permitiera lograr el objetivo. La intencionalidad de esta fase era que, de acuerdo con sus conocimientos previos y sus capacidades en la solución de problemas de matemáticas, la estudiante seleccionara la meta. Respetando las diferencias individuales se propuso una escala de metas: básico, medio y alto. Las metas de 
nivel básico contenían problemas sencillos y directos. El nivel medio proponía la solución de problemas utilizando métodos de factorización de polinomios. Por último, el nivel alto generó problemas de aplicación. En cada una de las metas debía ser seleccionada una valoración numérica. La meta y el puntaje seleccionado fueron almacenados en el ambiente m-learning (figura 2).

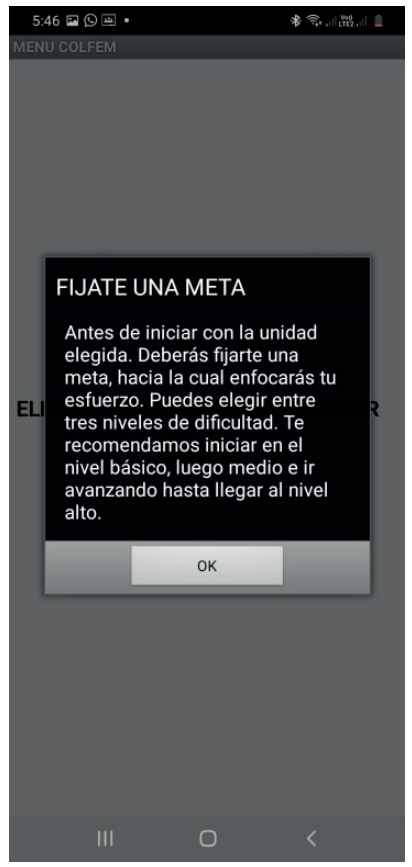

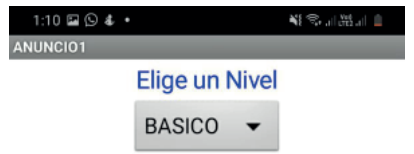

Ahora elige el puntaje que aspiras obtener ELIGE

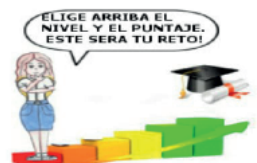

Elegiste el nive BASICO.Aprenderás a reconocer el caso de factorización que estas trabajando, y a resolver algunos ejercicios sencillos III 0
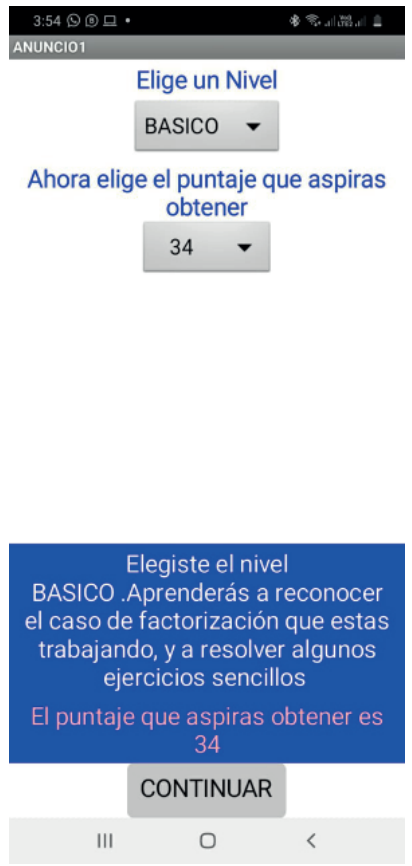

Figura 2. Formulación de metas de aprendizaje elaborada en la Plataforma App Inventor, de Google. Fuente: elaboración propia.

3. Elección juicio de autoeficacia: la estudiante debía realizar un juicio de autoeficacia, en la medida en que se cuestionaba sobre la selección de la meta de aprendizaje. Para esto, el ambiente ofreció cuatro alternativas: (a) me siento completamente segura de lograrlo, (b) me siento segura de lograrlo, (c) no estoy muy segura de lograrlo y, (d) me siento completamente segura de no lograrlo. Esta es una forma operativa que le permite al aprendiz monitorear su autoeficacia (Valencia-Vallejo et al., 2018).

4. Navegación en el ambiente: durante el desarrollo de esta aplicación la estudiante podía navegar libremente por el ambiente móvil. En la parte superior de cada unidad, el software presentaba un recuadro con la meta, con el fin de que la tuviera presente durante el proceso de aprendizaje. Esto le facilitaba el monitoreo. Durante la navegación por la estructura de ambiente m-learning, emergieron ventanas a través de las cuales se proponían retos mediante la resolución de problemas. De acuerdo con la respuesta, el andamiaje enviaba mensajes de retroalimentación positiva.

Se implementó una herramienta de autoevaluación en el ambiente m-learning para monitorear el aprendizaje en la estudiante. Una vez respondiera la pregunta, el software informaba si era correcta o incorrecta la respuesta y se emitía un mensaje para alentarla a seguir adelante o felicitarla por su avance. Al finalizar la prueba, el andamiaje mostraba el puntaje obtenido, lo comparaba con la meta definida e informaba si había alcanzado o no el objetivo propuesto. Lo anterior se acompañaba de frases motivadoras. La intencionalidad de 
esta herramienta era generar retos y experiencias positivas que activaran la eficacia personal de la estudiante.

\section{Prueba de estilo cognitivo.}

Para determinar el estilo cognitivo en la dimensión DIC, se utilizó el test de figuras enmascaradas (Embedded Figures Test) de Witkin, una prueba de percepción que requiere que el sujeto ubique una figura simple, vista previamente, dentro de una figura con un diseño complejo. La prueba contiene 18 figuras complejas. La tarea consiste en encontrar las figuras simples en un tiempo determinado. La prueba se aplica en forma grupal en un tiempo aproximado de 20 minutos. La confiabilidad del instrumento es alta ( $\alpha$ de Cronbach entre 0.82 - 0.90) (Witkin et al., 1977). Una versión del instrumento en línea fue aplicada en una prueba con estudiantes colombianos, la cual mostró un alfa de Cronbach de 0.847 (Hederich-Martínez, López-Vargas y Camargo-Uribe, 2016).

\section{Subescala de autoeficacia académica del instrumento MSLQ.}

Las estudiantes respondieron la subescala de autoeficacia académica del Cuestionario de Estrategias de Aprendizaje y Motivación (Motivated Strategies for Learning Questionnaire - MSLQ) (Pintrich, Smith, García y McKeachie, 1991). Este cuestionario de autoinforme planteaba ocho preguntas sobre autoeficacia para el aprendizaje y el desempeño. El test se respondió de acuerdo con una escala Likert de siete puntos $(1=\mathrm{No}$, nunca; 7 = Sí, siempre). Este test se ha aplicado en diferentes investigaciones realizadas con estudiantes colombianos y sus resultados mostraron altos índices de confiabilidad (Hederich-Martínez et al., 2016; López y Valencia, 2012; Valencia-Vallejo et al., 2018).

\section{Subescala de autoeficacia online.}

Se utilizó la subescala de autoeficacia en el aprendizaje online de la escala de autoeficacia y valor de aprendizaje (Online Learning Value and Self-Efficacy Scale - OLVSES). Es un cuestionario de autoinforme que planteaba cinco preguntas sobre autoeficacia, las cuales evaluaron la confianza de las estudiantes respecto de su capacidad para aprender a través de un ambiente con tecnologías móviles. El test se respondió de acuerdo con una escala Likert de siete puntos $(1=\mathrm{No}$, nunca; $7=S i$, siempre). La confiabilidad del instrumento es alta ( $\alpha$ de Cronbach $=0.87$ ) (Artino, 2010; Artino y McCoach, 2008; Zimmerman y Kulikowich, 2016).

\section{Logro de aprendizaje.}

El logro de aprendizaje se obtuvo del promedio de cuatro evaluaciones que presentaron las estudiantes de forma individual (una por cada módulo de estudio, contenidas en el ambiente m-learning). Todas las evaluaciones contenían cuatro problemas y las respuestas dadas fueron de selección múltiple.

\section{Procedimiento}

Para el desarrollo de la investigación se contactaron las directivas del Colegio Nacionalizado Femenino de Villavicencio, quienes aceptaron que las estudiantes del grado octavo participaran en el estudio. Posteriormente, fue presentada la propuesta de investigación, tanto a las estudiantes como a los profesores del área de Matemáticas, y se solicitó a los padres de las estudiantes su consentimiento para permitir la participación de las mismas, advirtiendo que los resultados serían confidenciales y con fines de investigación. Una vez firmados los formularios de consentimiento informado de los padres, se aplicó en forma grupal el test de figuras enmascaradas GEFT. Atendiendo al listado de las estudiantes, se procedió a asignarlas de forma aleatoria a dos grupos: uno que trabajaría con el ambiente $m$-learning con el andamiaje motivacional diseñado especialmente para esta investigación, y el otro grupo trabajó en el ambiente m-learning sin el andamiaje. Cada estudiante contaba con su correspondiente teléfono celular y la respectiva conectividad a internet.

Posteriormente, se realizó de forma presencial, a la totalidad de las estudiantes, una inducción frente a la instalación y funcionamiento del ambiente m-learning en sus respectivos teléfonos celulares. Las estudiantes interactuaron en el ambiente $m$-learning alrededor de cinco semanas. Una semana después de haber finalizado la experiencia, les fue aplicada la subescala de autoeficacia académica del instrumento MSLQ y la Escala de Autoeficacia online OLVSES, pruebas gestionadas a través de un formulario desarrollado en Google Drive. 


\section{Análisis de datos}

Se usó un análisis factorial de varianza Manova $2 \times 3$, que corresponde a los valores de la variable independiente y asociada del presente estudio. Previos a este procedimiento estadístico, las bases de datos fueron organizadas y validadas para garantizar la calidad de los resultados, utilizando el software Statistical Package for the Social Sciences, versión 24.

\section{Resultados}

\section{Estilo cognitivo en la dimensión DIC}

El promedio de la prueba GEFT de la muestra fue de 7.04, y la desviación estándar fue de 2.83. Sobre un puntaje máximo de 18, se obtuvo un valor mínimo de 3 puntos y un valor máximo de 14 puntos. Las estudiantes se agruparon en DC, intermedios e IC, con base en los terciles del puntaje GEFT, de forma tal que se identificaron tres rangos de puntajes: (a) 20 estudiantes DC (primer tercil), (b) 15 estudiantes intermedios (segundo tercil), y (c) 21 estudiantes IC (tercer tercil).

\section{Autoeficacia académica}

El promedio de la subescala de autoeficacia académica fue de 5.80, y la desviación estándar fue de 0.70. Sobre un puntaje máximo de 7 puntos, el valor mínimo obtenido fue 4.13 y el máximo de 6.88. En el presente estudio, la subescala presentó una fiabilidad de 0.72 .

\section{Autoeficacia online}

La media de la subescala de autoeficacia online fue de 5.60, y la desviación estándar fue de 0.97. Sobre un puntaje máximo de 7 , se obtuvo un valor mínimo de 3 puntos y un valor máximo de 7 puntos. La subescala presentó un alfa de Cronbach de 0.84.

\section{Logro de aprendizaje}

El promedio de logro de aprendizaje en las estudiantes fue de 3.53, y la desviación estándar fue de 0.66. Sobre un puntaje máximo de 5 , el logro más bajo fue de 2.50 y el más alto fue de 4.69. Las evaluaciones presentaron una fiabilidad de 0.79.

\section{Análisis factorial multivariado}

Se realizó un análisis factorial Manova 2×3. En la tabla 1 se presenta el resumen de los estadísticos descriptivos de los grupos de estudiantes que trabajaron en el ambiente $m$-learning con andamiaje y sin andamiaje, teniendo en cuenta el estilo cognitivo.

Tabla 1

Promedios obtenidos (DE) en las variables dependientes

\begin{tabular}{clcccc}
\hline $\begin{array}{c}\text { Ambiente } \\
\text { m-learning }\end{array}$ & \multicolumn{1}{c}{ Estilo cognitivo } & Núm. & $\begin{array}{c}\text { Logro de } \\
\text { aprendizaje }\end{array}$ & $\begin{array}{c}\text { Autoeficacia } \\
\text { online }\end{array}$ & $\begin{array}{c}\text { Autoeficacia } \\
\text { académica }\end{array}$ \\
\hline & Dependiente de campo & 10 & $3.22(0.66)$ & $4.90(1.12)$ & $5.68(0.73)$ \\
$\begin{array}{c}\text { Sin andamiaje } \\
\text { de autoeficacia }\end{array}$ & Intermedio & 7 & $3.35(0.72)$ & $4.97(1.22)$ & $5.27(0.64)$ \\
& Independiente de campo & 11 & $3.38(0.54)$ & $5.75(0.87)$ & $5.98(0.66)$ \\
& Total & 28 & $3.31(0.61)$ & $5.25(1.10)$ & $5.69(0.71)$ \\
$\begin{array}{l}\text { Con andamiaje } \\
\text { de autoeficacia }\end{array}$ & Intermedio & 10 & $3.38(0.72)$ & $5.72(0.77)$ & $5.85(0.75)$ \\
& Independiente de campo & 10 & $4.00(0.55)$ & $5.90(0.50)$ & $5.63(0.58)$ \\
& Total & 28 & $3.77(0.64)$ & $5.99(0.66)$ & $5.92(0.68)$ \\
\hline
\end{tabular}

Fuente: elaboración propia. 
Los resultados de los contrastes multivariados del análisis Manova indican que el ambiente m-learning (Wilks ' $\Lambda=0.74, \quad F(3,47)=5.57$, $\left.p=0.002, \eta^{2}=0.26\right)$ y la interacción entre el ambiente m-learning y el estilo cognitivo en la dimensión DIC (Wilks ' $\Lambda=0.75, \quad F(6,94)=2.41, p=0.033$, $\left.\eta^{2}=0.13\right)$ afectaron significativamente las variables dependientes, es decir, el logro de aprendizaje, la autoeficacia académica y la autoeficacia online. El estilo cognitivo no afectó de forma significativa ninguna de las variables dependientes (Wilks $\left.{ }^{\prime} \Lambda=0.84, F(6,94)=1.41, p=0.220, \eta^{2}=0.08\right)$. Los tamaños del efecto multivariantes fueron moderados. Los resultados indican que existe una interacción significativa entre el ambiente m-learning y el estilo cognitivo $\left(F(2,49)=5.81, p=0.005, \eta^{2}=0.19\right)$ solo en la autoeficacia académica de las estudiantes.

Se evidenciaron diferencias significativas en el logro de aprendizaje debido al efecto del ambiente m-learning, en favor de las estudiantes que utilizaron el andamiaje $(F(1,49)=8.36, p=0.006$, $\left.\eta^{2}=0.15\right)$. También se presentaron diferencias significativas en la autoeficacia online por efecto del ambiente m-learning, a favor de las estudiantes que interactuaron con el andamiaje motivacional $\left(F(1,49)=12.16, p=0.001, \eta^{2}=0.20\right)$. Los hallazgos revelaron que no existen diferencias significativas en la autoeficacia académica por efecto del ambiente m-learning $\left(F(1,49)=3.49, p=0.068, \eta^{2}=0.07\right)$.

Frente a la variable de estilo cognitivo en la dimensión DIC, los resultados mostraron que no existen diferencias significativas en ninguna de las variables dependientes. Es decir, no se registraron diferencias significativas en el logro de aprendizaje $\left(F(2,49)=2.63, \quad p=0.082, \quad \eta^{2}=0.10\right)$, en la autoeficacia académica $(F(2,49)=0.09, p=0.917$, $\left.\eta^{2}<0.00\right)$ y tampoco en la autoeficacia online $(F(2$, 49) $=1.96, p=0.152, \eta^{2}=0.07$ ).

Los resultados post hoc de Bonferroni indicaron que en el ambiente m-learning con andamiaje motivacional se presentaron diferencias estadísticamente significativas en la percepción de autoeficacia online $(p<0.05)$ entre estudiantes intermedias $(M=6.45, S D=0.43)$ y estudiantes IC $(M=5.63, S D=0.58)$. Se observaron diferencias estadísticamente significativas en la autoeficacia online $(p<0.05)$ entre estudiantes intermedias de campo que interactuaron con el ambiente $m$-learning con andamiaje $(M=6.44, S D=0.43)$ y los intermedios que interactuaron con el ambiente $m$-learning $\sin$ andamiaje $(M=5.27, S D=0.64)$ (figura 5).

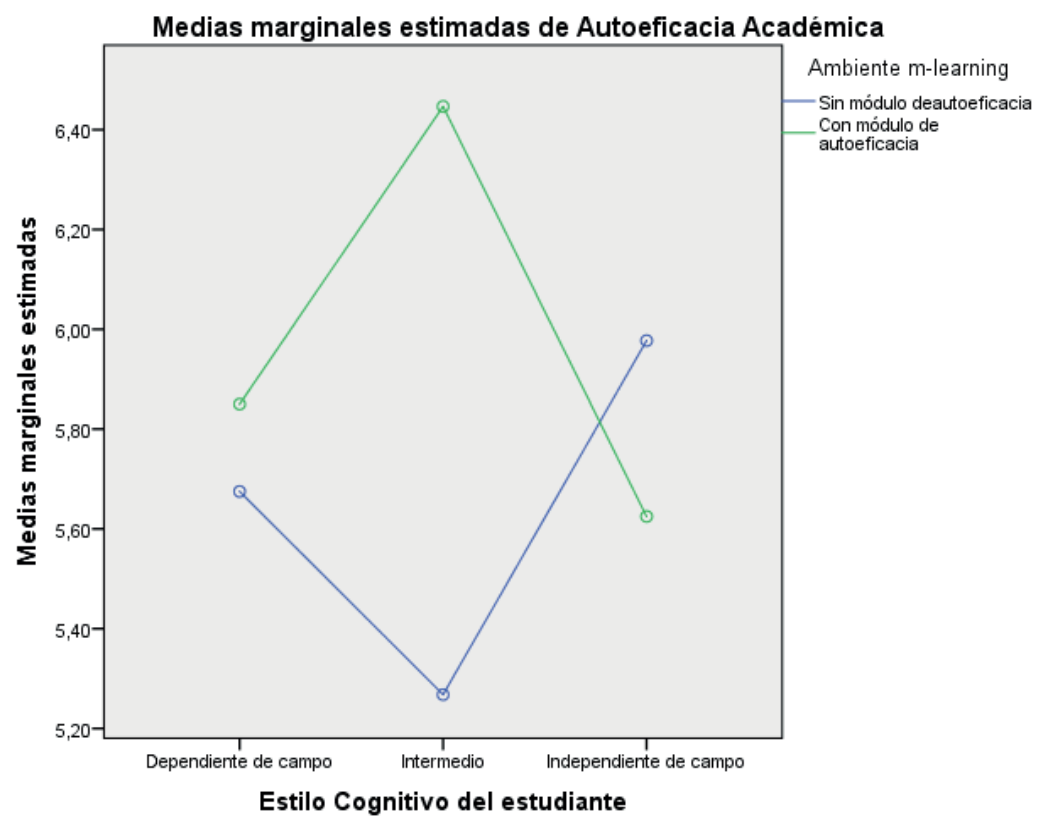

Figura 5. Efecto del estilo cognitivo y el ambiente $m$-learning sobre la autoeficacia académica. Fuente: elaboración propia. 


\section{Discusión}

De los resultados del estudio se puede concluir que el uso de andamiajes motivacionales en interacción con el estilo cognitivo del estudiante, favorece de forma significativa la autoeficacia académica. De igual forma, los hallazgos revelaron que la inclusión de un andamiaje motivacional conduce a favorecer la autoeficacia online y el logro de aprendizaje. En otras palabras, la implementación de un andamiaje, diseñado para favorecer la autoeficacia, puede ayudar a reducir de forma positiva las diferencias individuales en el logro de aprendizaje de los estudiantes de diferente estilo cognitivo en la dimensión DIC. En este orden de ideas, y teniendo en cuenta las preguntas de investigación que orientaron esta investigación, se presenta la siguiente discusión.

Frente a la primera pregunta de investigación, los resultados del estudio permitieron dar cuenta de que el uso de un andamiaje de autoeficacia dentro de la estructura de un ambiente m-learning, afectó de forma positiva la autoeficacia online y el logro de aprendizaje del estudiante. En esta medida, la autoimposición de una meta de aprendizaje por parte del estudiante y el monitorear el logro de la misma, hizo posible que ganara confianza sobre sus capacidades para obtener el logro deseado. El andamiaje tuvo como objetivo apoyar al estudiante en el desempeño académico respetando su ritmo de aprendizaje y sus diferencias individuales.

La imposición de metas de forma creciente posibilitó que el estudiante percibiera experiencias de aprendizaje positivas que lo llevaron al logro de sus propósitos, incrementando de forma positiva su percepción de autoeficacia tanto académica como online. Esta situación condujo a que el aprendiz, en la medida en que gane confianza en sus conocimientos, se propone metas más exigentes y realistas de acuerdo con sus capacidades. Sin lugar a dudas, esta estrategia pedagógica o didáctica fue una forma de estimular el aprendizaje a través de tecnologías móviles y también se constituyó en una herramienta potencial de trabajo que favoreció la autorregulación del aprendizaje, habilidad de gran interés por la comunidad académica de las TIC, por cuanto se preparó a los aprendices para asumir el reto de la formación apoyados con ambientes de aprendizaje basados en la web.
El uso de la herramienta de autoevaluación de aprendizajes y los mensajes motivacionales dispuestos en el andamiaje, procuró que las estudiantes se esforzaran y fueran persistentes en el logro de las metas de desempeño deseadas. En este aspecto, la retroalimentación positiva fue importante para mejorar el sentido de autoeficacia. Estos hallazgos constituyen evidencia empírica en este campo de investigación y apoya los resultados de algunos estudios que relacionan el uso de andamiajes motivacionales para favorecer la autoeficacia, el desempeño y la autorregulación del aprendizaje en ambientes computacionales (López et al., 2014; López y Triana, 2013; López y Valencia, 2012; Valencia-Vallejo et al., 2018). En este ámbito de trabajo, los hallazgos de la investigación apoyaron los resultados de los estudios de Razzaq et al. (2018), Yang (2012) y Yorganci (2017) sobre el desarrollo de la autoeficacia y el mejoramiento del logro académico en ambientes m-learning.

Con respecto a la segunda pregunta de investigación, los resultados mostraron que no existen diferencias en el logro de aprendizaje y la autoeficacia, tanto académica como online, entre las estudiantes que participaron en la investigación obedeciendo a su estilo cognitivo en la dimensión DIC. Es decir, las estudiantes IC, intermedias y DC lograron aprendizajes y percepciones de eficacia académica y online equivalentes a aprender contenidos matemáticos a través de un ambiente m-learning.

De acuerdo con los resultados de la investigación, los ambientes m-learning, que incorporan dentro de su estructura andamiajes de tipo motivacional, estarían en capacidad de favorecer un aprendizaje más equitativo, atendiendo las diferencias individuales del aprendiz. En consecuencia, un andamiaje motivacional puede ser una estrategia pedagógica o didáctica para favorecer el logro de aprendizaje de aquellas estudiantes con dificultades en el área de las Matemáticas, más específicamente, en las estudiantes DC, quienes de manera sistemática presentan bajos resultados de aprendizaje y actitudes menos favorables en el aprendizaje de estas y las ciencias, en comparación con las estudiantes IC (Hederich-Martínez y Camargo-Uribe, 2015; López et al., 2011).

Al mismo tiempo, el uso de andamiajes motivacionales constituye una herramienta que favorece la percepción de autoeficacia académica 
en el aprendizaje de las matemáticas en estudiantes DC, quienes probablemente presentan un déficit motivacional de acuerdo con el campo de la investigación de la autorregulación del aprendizaje (López et al., 2011; Valencia-Vallejo, López-Vargas y Sanabria-Rodríguez, 2019). También estarían en capacidad de apoyar a estudiantes DC en el uso efectivo y eficaz de las tecnologías de la información en procesos de aprendizaje individual, quienes prefieren la navegación en grupo o guiados por otra persona. El uso del andamiaje puede llegar a ser una estrategia interesante para este tipo de sujetos, en la medida en que los apoya en el desarrollo de trabajos de forma individual, para así generar autonomía en su proceso de aprendizaje.

Aunque estos resultados no son concluyentes, son prometedores en esta línea de investigación, debido a que el diseño de andamiajes motivacionales puede llegar a ser interesante, en tanto favorecen el aprendizaje de las estudiantes DC, resultados que sugieren profundizar sobre el diseño e implementación de los mismos en escenarios en m-learning (Huertas, López y Sanabria, 2017; LópezVargas et al., 2017; Valencia-Vallejo et al., 2018); además de empezar a utilizar estas herramientas de trabajo en estudiantes de secundaria, como una forma de favorecer la autonomía en el aprendizaje y prepararlos para asumir el reto de la educación superior.

Con respecto a la tercera pregunta de investigación, los resultados sugieren una interacción significativa entre el ambiente m-learning y el estilo cognitivo en la dimensión DIC. De acuerdo con los resultados, una posible forma de favorecer, de forma positiva, la percepción de autoeficacia académica en las estudiantes con diferente estilo cognitivo, es la implementación de andamiajes dentro de la estructura de los ambientes m-learning. Este hallazgo es prometedor en la medida en que estudiantes de educación secundaria pueden ver en las tecnologías móviles una herramienta que facilite su proceso de aprendizaje respetando sus diferencias individuales. De esta forma, estarían en capacidad de utilizar sus teléfonos inteligentes no solo como un escenario de juego o de redes sociales, sino como escenarios potenciales de aprendizaje en donde pueden acceder a contenidos y materiales de estudio desde cualquier lugar $y$ en cualquier tiempo, dadas sus características de portabilidad y conectividad.
Así las cosas, sería recomendable impulsar en la educación secundaria, el uso de ambientes m-learning que incorporen andamiajes motivacionales en los cuales se respeten las diferencias individuales de los estudiantes. El uso de esta estrategia pedagógica puede inducir a los aprendices a usar las tecnologías móviles como escenario de aprendizaje en donde puedan lograr los desempeños deseados. Sin embargo, los resultados de investigación en este ámbito aún no son concluyentes y se hace necesario seguir investigando al respecto, de tal manera que sea posible comprender y explicar la conducta de los estudiantes cuando interactúan con ambientes de aprendizaje apoyados con tecnologías móviles.

Una de las limitaciones presentes al desarrollar la investigación fue el tamaño de la muestra. Seguramente, un mayor número de participantes habría permitido una mayor generalización de los resultados. Igualmente, hubiese sido importante la participación de estudiantes de ambos géneros, ya que la presente investigación se realizó en una institución femenina, situación que no permitió tomar una muestra representativa por género. Además, la subjetividad en las respuestas proporcionadas por las participantes fue un factor limitante de los cuestionarios de autoinforme, como fue el caso del instrumento que se utilizó para medir la autoeficacia. Sería conveniente usar otros indicadores que permitan evidenciar estas variables de manera objetiva.

\section{Referencias}

Aesaert, K., Voogt, J., Kuiper, E., \& Van-Braak, J. (2017). Accuracy and Bias of ICT Self-Efficacy: An Empirical Study into Students' over and Underestimation of their ICT Competences. Computers in Human Behavior, 75, 92-102. doi: 10.1016/j.chb.2017.05.010

Alias, N. (2012). Design of a Motivational Scaffold for the Malaysian e-Learning Environment. Educational Technology and Society, 15(1), 137-151. Retrieved from https://eric. ed.gov/?id=EJ979457

Artino, A. R. (2010). Online or Face-to-Face Learning? Exploring the Personal Factors that Predict Students' Choice of Instructional 
Format. Internet and Higher Education, 13(4), 272-276. doi: 10.1016/j.iheduc.2010.07.005

Artino, A. R., \& McCoach, D. B. (2008). Development and Initial Validation of the Online Learning Value and Efficacy Scale. Journal of Educational Computing Research, 38(3), 279-303. doi: 10.2190/EC.38.3.C

Bandura, A. (1997). Self-Efficacy: The Exercise of Control. New York: Freeman.

Belland, B., Kim, C., \& Hannafin, M. (2013). A Framework for Designing Scaffolds that Improve Motivation and Cognition. Educational Psychologist, 48(4), 243-270. doi: 10.1080/00461520.2013.838920

Campbell, D. y Stanley, J. (2005). Diseños experimentales y cuasiexperimentales en la investigación social (9. a ed.). Buenos Aires: Amorrortu.

Chen, S., \& Macredie, R. (2001). Cognitive Styles and Hypermedia Navigation: Development of a Learning Model. Journal of the American Society for Information Science and Technology, 53(1), 3-15. doi: 10.1002/asi.10023

Chou, H. W. (2001). Influences of Cognitive Style and Training Method on Training Effectiveness. Computers and Education, 37(1), 11-25. https:// doi.org/10.1016/S0360-1315(01)00028-8

Compeau, D. R., \& Higgins, C. A. (1995). Computer Self-Efficacy: Development of a Measure and Initial Test. Management Information Systems Quarterly, 19(2), 189-211. doi: 10.2307/249688

DeTure, M. (2004). Cognitive Style and SelfEfficacy: Predicting Student Success in Online Distance Education. American Journal of Distance Education, 18(1), 21-38. https://doi. org/10.1207/s15389286ajde1801_3

D’Mello, S., Lehman, B., \& Graesser, A. (2011). A Motivationally Supportive Affect-Sensitive Autotutor. In R. Calvo, \& S. D'Mello (Eds.), New Perspectives on Affect and Learning Technologies (pp.113-126). New York: Springer.

Duffy, M. C., \& Azevedo, R. (2015). Motivation Matters: Interactions between Achievement Goals and Agent Scaffolding for Self-Regulated Learning within an Intelligent Tutoring System. Computers in Human Behavior, 52, 338-348. https://doi.org/10.1016/j.chb.2015.05.041
Handal, B., \& Herrington, T. (2004). On being dependent and independent in computer based learning environments. e-Journal of Instructional Science and Technology, (7)2. Retrieved from http://files.eric.ed.gov/fulltext/ EJ850352.pdf

Hatlevik, O., Throndsen, I., Loi, M., \& Gudmundsdottir, G. (2018). Students' ICT Self-Efficacy and Computer and Information Literacy: Determinants and Relationships. Computers \& Education, 118, 107-119. doi: 10.1016/j.compedu.2017.11.011

Hederich-Martínez, C., \& Camargo-Uribe, A. (2015). Cognitive Style and Educational Performance. The Case of Public Schools in Bogotá, Colombia. Educational Psychology, 36(4), 719737. doi: 10.1080/01443410.2015.1091916

Hederich-Martínez, C., López-Vargas, O., \& Camargo-Uribe, A. (2016). Effects of the Use of a Flexible Metacognitive Scaffolding on SelfRegulated Learning During Virtual Education. International Journal of Technology Enhanced Learning, 8(3/4), 199-216. doi: 10.1504/ IJTEL.2016.10002201

Huang, Y. M., Jeng, Y. L., \& Huang, T. C. (2009). An Educational Mobile Blogging System for Supporting Collaborative Learning. Educational Technology \& Society, 12(2), 163175. Retrieved from https://www.learntechlib. org/p/75319/

Huertas, A., López, O., \& Sanabria, L. (2017). Influence of a Metacognitive Scaffolding for Information Search in B- Learning Courses on Learning Achievement and its Relationship with Cognitive and Learning Style. Journal of Educational Computing Research, 55(2), 1-25. doi: 10.1177/0735633116656634

Keller, J. (2010). Motivational Design for Learning and Performance. The ARCS model approach. Boston: Springer Science \& Business Media. doi: 10.1007/978-1-4419-1250-3

Kim, M. C., \& Hannafin, M. J. (2011). Scaffolding Problem Solving in Technology-Enhanced Learning Environments (TELEs): Bridging Research and Theory with Practice. Computers \& Education, 56(2), 403-417. doi: 10.1016/j. compedu.2010.08.024 
Kukulska-Hulme, A. G., \& Traxler, J. (2005). Mobile Learning. A Handbook for Educators and Trainers. New York: Routedge Falmer.

Lehmann, T., Hähnlein, I., \& Ifenthaler, D. (2014). Cognitive, Metacognitive and Motivational Perspectives on Preflection in Self-Regulated Online Learning. Computers in Human Behavior, 32, 313-323. doi: 10.1016/j. chb.2013.07.051

López, O., Hederich, C. y Camargo, A. (2011). Estilo cognitivo y logro académico. Educación y Educadores, 14(1), 67-82. doi: 10.5294/ edu.2011.14.1.4

López, O., Sanabria, L. y Sanabria, M. (2014). Logro de aprendizaje en ambientes computacionales. Autoeficacia, metas y estilo cognitivo. Psicología desde el Caribe, 31(3), 475-494. http://dx.doi.org/10.14482/psdc.31.3.5366

López, O. y Triana, S. (2013). Efecto de un activador computacional de autoeficacia sobre el logro de aprendizaje en estudiantes de diferente estilo cognitivo. Revista Colombiana de Educación, 64(1), 225-244. doi: 10.17227/01203916.64rce225.244

López, O. y Valencia, N. (2012). Diferencias individuales en el desarrollo de la autoeficacia y el logro académico: el efecto de un andamiaje computacional. Acta Colombiana de Psicología, 15(2), 29-41. Recuperado de http://www.redalyc.org/articulo.oa?id=7982 5836001

López-Vargas, O., Duarte-Suárez, L., \& IbáñezIbáñez, J. (2017). Teacher's Computer SelfEfficacy and its Relationship with Cognitive Style and TPACK. Improving Schools, 20(3), 264-277. doi: 10.1177/1365480217704263

López-Vargas, O., Ibáñez-Ibáñez, J., \& RacinesPrada, O. (2017). Students' Metacognition and Cognitive Style and their Effect on Cognitive Load and Learning Achievement. Educational Technology \& Society, 20(3), 145-157. Recuperado de https://eric. ed.gov/?id=EJ1147009

Papastergiou, M., Gerodimos, V., \& Antoniou, P. (2011). Multimedia Blogging in Physical Education: Effects of Student Knowledge and ICT Self-Efficacy. Computers \&
Education, 57(3), 1998-2010. doi: 10.1016/j. compedu.2011.05.006

Pintrich, P., Smith, D., García, T., \& McKeachie, W. (1991). A Manual for the Use of the Motivated Strategies for Learning Questionnaire (MSLQ). Ann Arbor, MI: National Center for Research to Improve Postsecondary Teaching and Learning.

Razzaq, A., Samiha, Y., \& Anshari, M. (2018). Smartphone Habits and Behaviors in Supporting Students Self-Efficacy. International Journal of Emerging Technologies in Learning, 13(2), 94-109. http://dx.doi.org/10.3991/ijet. v13i02.7685

Tsai, C. C., Chuang, S. C., Liang, J. C., \& Tsai, M. J. (2011). Self-efficacy in internet-based learning environments: A literature review. Educational Technology \& Society, 14(4), 222-240. Retrieved from https://www.jstor.org/stable/ jeductechsoci.14.4.222? seq=1

Tsai, M. J., \& Tsai, C. C. (2010). Junior High School Students' Internet Usage and Self-Efficacy: A Re-Examination of the Gender Gap. Computers \& Education, 54(4), 1182-1192. Retrieved from https://www.learntechlib.org/p/67427/

Valencia-Vallejo, N., López-Vargas, O., \& SanabriaRodríguez, L. (2018). Effect of a Motivational Scaffolding on e-Learning Environments: SelfEfficacy, Learning Achievement, and Cognitive Style. Journal Educators On-line, 15(1), 1-14. Retrieved from https://files.eric.ed.gov/fulltext/ EJ1168944.pdf

Valencia-Vallejo, N., López-Vargas, O., \& SanabriaRodríguez, L. (2019). Effect of a Metacognitive Scaffolding on Self-Efficacy, Metacognition, and Achievement in e-Learning Environments. Knowledge Management \& ELearning, 11(1), 1-19. https://doi.org/10.34105/j. kmel.2019.11.001

Vygotsky, L. (1978). Mind in society: The Development of Higher Mental Process. Cambridge: Harvard University Press.

Witkin, H., Moore, C., Goodenough, D., \& Cox, P. (1977). Field-Dependent and Field-Independent Cognitive Styles and their Educational Implications. Review of Educational Research, 47(1), 1-64. doi: 10.3102/00346543047001001 
Wood, D., Bruner, J., \& Ross, G. (1976). The Role of Tutoring in Problem Solving. Journal of Child Psychology and Psychiatry, and Allied Disciplines, 17(2), 89-100. doi: 10.1111/ j.1469-7610.1976.tb00381.x

Yang, S. H. (2012). Exploring College Students' Attitudes and Self-Efficacy of Mobile Learning. Turkish Online Journal of Educational Technology, 11(4), 148-154. Retrieved from https://eric.ed.gov/?id=EJ989264

Yorganci, S. (2017). Investigating Students' SelfEfficacy and Attitudes towards the Use of Mobile Learning. Journal of Education and Practice, 8(6), 181-185. Retrieved from https:// files.eric.ed.gov/fulltext/EJ1133019.pdf
Zhang, M., \& Quintana, C. (2012). Scaffolding Strategies for Supporting Middle School Students' Online Inquiry Processes. Computers and Education, 58(1), 181-196. doi: 10.1016/j. compedu.2011.07.016

Zheng, L., Li, X., \& Chen, F. (2016). Effects of a Mobile Self-Regulated Learning Approach on Students' Learning Achievements and SelfRegulated Learning Skills. Innovations in Education and Teaching International, 55(6), 1-9. doi: 10.1080/14703297.2016.1259080

Zimmerman, W.A., \& Kulikowich, J.M. (2016). Online Learning Self-Efficacy in Students with and without Online Learning Experience. American Journal of Distance Education, 30(3), 180-191. doi: 10.1080/08923647.2016.1193801

Para citar este artículo / To cite this article / Para citar este artigo: López-Vargas, O., OrtizVásquez, J. e Ibáñez-Ibáñez, J. (2020). Autoeficacia y logro de aprendizaje en estudiantes con diferente estilo cognitivo en un ambiente m-learning. Pensamiento Psicológico, 18(1), 71-85. doi:10.11144/Javerianacali.PPSI18-1.alae 